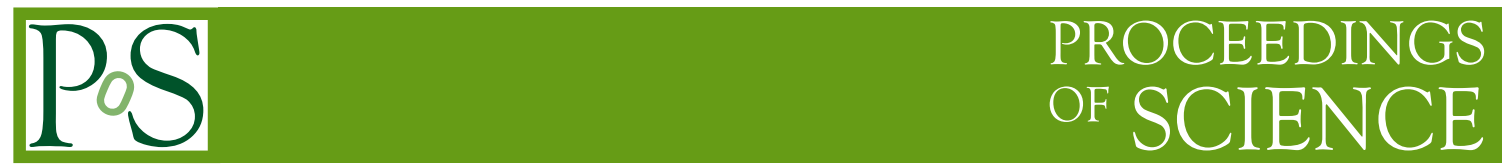

\title{
Charged current pi-plus production from the K2K Scibar
}

\author{
LISA WHITEHEAD*i \\ Brookhaven National Laboratory \\ E-mail: 1whitehead@bnl.gov
}

Single charged pion production in charged-current muon neutrino interactions with carbon is studied using data collected in the $\mathrm{K} 2 \mathrm{~K}$ long-baseline neutrino experiment. Our measurement of the cross section relative to that of charged-current quasi-elastic is consistent with results of a previous experiment and our prediction based on the Rein and Sehgal model.

10th International Workshop on Neutrino Factories, Super beams and Beta beams

June 30 - July 52008

Valencia, Spain

\footnotetext{
*Speaker.

${ }^{\dagger}$ for the K2K Collaboration
} 


\section{Introduction}

Charged-current single $\pi^{+}$production via resonance $\left(\mathrm{CC} 1 \pi^{+}\right)$in $v_{\mu}$ interactions is the largest background to charged-current quasielastic (CCQE) interactions in the region of neutrino energy around $1 \mathrm{GeV}$. A better understanding of the $\mathrm{CC} 1 \pi^{+}$cross section is important for precision neutrino oscillation studies.

The K2K experiment [1] is a long-baseline neutrino oscillation experiment located in Japan in which a beam of muon neutrinos is produced at KEK and detected $250 \mathrm{~km}$ away with the SuperKamiokande detector [2]. The mean energy of the neutrinos is $1.3 \mathrm{GeV}$. The SciBar detector [3, 那 is one of the near detectors for K2K located at KEK. It is a fully active scintillator detector consisting of 14,848 scintillating bars. A wavelength shifting fiber is inserted through each bar to guide the scintillation light to 64-channel multianode photomultiplier tubes. Groups of bars are arranged vertically or horizontally to make $x$ - or $y$-planes. One layer consists of one $x$-plane and one $y$-plane; there are 64 layers total. Each plane is $3 \mathrm{~m} \times 3 \mathrm{~m}$, and the detector extends $1.7 \mathrm{~m}$ in the beam direction. The SciBar detector took data as part of the K2K experiment from October 2003 until November 2004.

Resonant single pion production in K2K's Monte Carlo (MC) is simulated based on the model of Rein and Sehgal [5], which is commonly used in neutrino interaction generators for oscillation experiments. We set the axial-vector mass, $M_{A}$, for single pion production to be $1.1 \mathrm{MeV} / \mathrm{c}^{2}$. Charged-current (CC) coherent $\pi^{+}$production is not included in our model based on a measurement made with the SciBar detector [6]. More detailed descriptions of the neutrino interaction simulation used by $\mathrm{K} 2 \mathrm{~K}$ can be found elsewhere [1, 7].

\section{Cross Section Measurement}

To select CC events, we search for tracks starting in the fiducial volume of SciBar that are matched with a track or hits in the first layer of the Muon Range Detector (MRD), located just downstream of SciBar. The MRD matching requirement imposes a muon momentum $\left(p_{\mu}\right)$ threshold of $450 \mathrm{MeV} / c$. The sample of events in which a SciBar-MRD track is found is our CC sample. According to MC simulation, $96 \%$ of the events in the $\mathrm{CC}$ sample are true $\mathrm{CC}$ interactions. The muon momentum resolution is $90 \mathrm{MeV} / \mathrm{c}$, and the muon angle $\left(\theta_{\mu}\right)$ resolution is 1.4 degrees. We count the number of tracks at the event vertex, where the vertex is defined as the upstream endpoint of the SciBar-MRD track. We consider only 1- and 2-track events. 2-track events are classified as quasi-elastic-like (QE-like) or nonQE-like, based on kinematics. 2-track nonQE-like events are classified as pion-like or proton-like using a particle identification variable based on the measured $\mathrm{dE} / \mathrm{dx}$. Thus there are four samples of events: 1-track, 2-track QE, 2-track nonQE pion, and 2-track nonQE proton. These samples differ in their relative contributions from CCQE, CC1 $\pi^{+}$, and other types of neutrino interactions.

We normalize our measurement of the $\mathrm{CC} 1 \pi^{+}$cross section to the CCCQE cross section to reduce the impact of neutrino flux uncertainties. We measure both an overall cross section ratio and an energy-dependent cross section ratio. Data and MC in the four event samples described above are binned in $p_{\mu}$ bins of size $0.2 \mathrm{GeV} / c$ and $\theta_{\mu}$ bins of size 10 degrees. We perform a maximum likelihood fit to determine the cross sections of $\mathrm{CC} 1 \pi^{+}$and $\mathrm{CCQE}$ relative to the MC predictions. 
From the MC prediction and the results of the fit, the observed $\mathrm{CC} 1 \pi^{+}$to $\mathrm{CCQE}$ cross section ratio can be extracted. Note that we do not identify $\mathrm{CC} 1 \pi^{+}$interactions on an event-by-event basis; we measure the rate of the $\mathrm{CC} 1 \pi^{+}$interaction relative to what is predicted by our MC. A detailed description of the fitting can be found elsewhere [8].

The dominant systematic uncertainties come from the uncertainties in modeling interactions of protons and pions in the nucleus and the uncertainty in the value of the axial-vector mass for the CCQE interaction. For the energy-dependent measurement, uncertainty in the neutrino energy spectrum measurement is also a significant source.

The results for both the energy-independent and energy-dependent measurement are shown in Table 1. The MC prediction for the total cross section ratio is 0.740 . Figure 1 shows the results of the energy-dependent measurement compared to the MC prediction. Figure 2 shows the result of the energy-dependent measurement compared to results from the ANL bubble chamber experiment [9, 10].

\begin{tabular}{|c|c|}
\hline $\begin{array}{c}\text { Energy Range } \\
(\mathrm{GeV})\end{array}$ & $\begin{array}{l}\text { Cross Section Ratio } \\
\qquad R_{k}=\frac{\sigma_{k}^{C C 1 \pi^{+}}}{\sigma_{k}^{C C Q E}} \\
\end{array}$ \\
\hline$>0.00$ & $0.734 \pm 0.086(\text { fit })_{-0.103}^{+0.076}(\text { nucl })_{-0.073}^{+0.079}$ (syst) \\
\hline $0.00-1.35$ & $0.402 \pm 0.111$ (fit) $)_{-0.071}^{+0.079}(\mathrm{nucl})_{-0.092}^{+0.131}$ (syst) \\
\hline $1.35-1.72$ & $1.022 \pm 0.167$ (fit $)_{-0.217}^{+0.072}(\text { nucl })_{-0.139}^{+0.107}$ (syst) \\
\hline $1.72-2.22$ & $1.007 \pm 0.214(\text { fit })_{-0.065}^{+0.209}(\text { nucl })_{-0.173}^{+0.241}$ (syst) \\
\hline$>2.22$ & $1.450 \pm 0.324(\text { fit })_{-0.272}^{+0.200}(\text { nucl })_{-0.480}^{+0.330}$ (syst) \\
\hline
\end{tabular}

Table 1: Cross Section Ratio

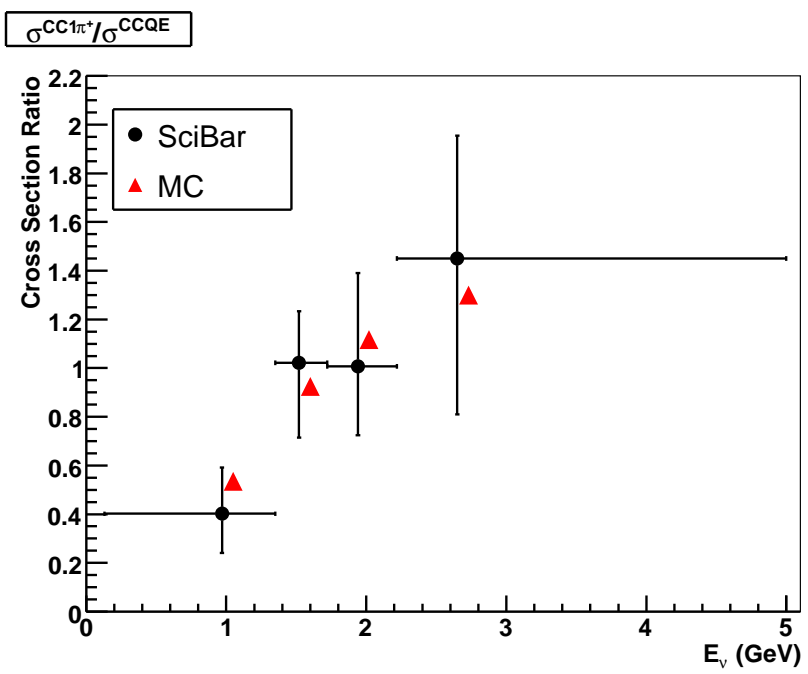

Figure 1: Comparison of the results with the MC prediction. 


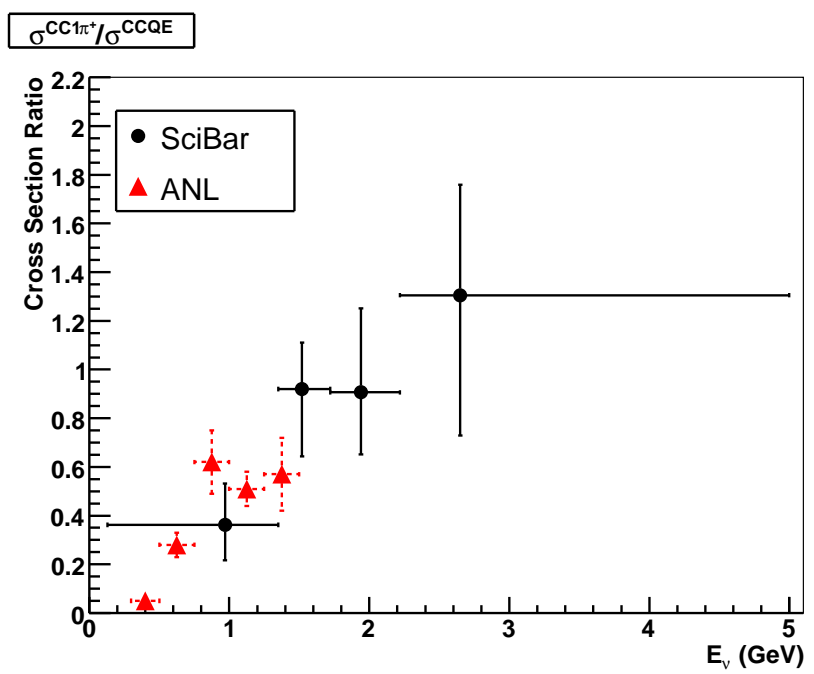

Figure 2: Comparison of the results with the results of the ANL experiment [9, 10].

\section{Conclusion}

A measurement of the $\mathrm{CC} 1 \pi^{+}$to $\mathrm{CCQE}$ cross section ratio was performed using data collected by the SciBar detector as part of the $\mathrm{K} 2 \mathrm{~K}$ experiment. The result is consistent with the MC prediction based on the Rein and Sehgal model and with results from the ANL bubble chamber experiment. The results were recently published [8], and a more detailed description of the analysis is found in that reference.

\section{References}

[1] M. H. Ahn et al. [K2K Collaboration], Phys. Rev. D 74, 072003 (2006) [arXiv:hep-ex/0606032].

[2] Y. Fukuda et al., Nucl. Instrum. Meth. A 501, 418 (2003).

[3] K. Nitta et al., Nucl. Instrum. Meth. A 535147 (2004).

[4] S. Yamamoto et al., IEEE Trans. Nucl. Sci. 522992 (2005).

[5] D. Rein and L. M. Sehgal, Annals Phys. 133, 79 (1981).

[6] M. Hasegawa et al. [K2K Collaboration], Phys. Rev. Lett. 95, 252301 (2005) [arXiv:hep-ex/0506008].

[7] Y. Hayato, Nucl. Phys. Proc. Suppl. 112, 171 (2002).

[8] A. Rodriguez et al. [K2K Collaboration], Phys. Rev. D 78, 032003 (2008) [arXiv:0805.0186 [hep-ex]].

[9] G. M. Radecky et al., Phys. Rev. D 25, 1161 (1982) [Erratum-ibid. D 26, 3297 (1982)].

[10] S. J. Barish et al., Phys. Rev. D 16, 3103 (1977). 